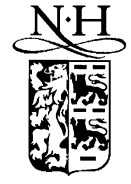

ELSEVIER

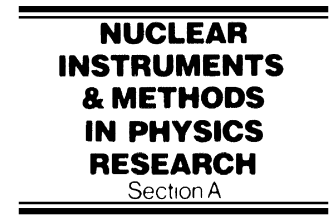

www.elsevier.nl/locate/nima

\title{
Low-dose radiation damage and recovery of undoped BGO crystals
}

\author{
K.C. Peng ${ }^{\mathrm{a}}$, R.S. $\mathrm{Lu}^{\mathrm{a}}$, K. Ueno ${ }^{\mathrm{a}, *}$, C.H. Wang ${ }^{\mathrm{b}}$, M.Z. Wang ${ }^{\mathrm{a}}$, \\ F.I. Chou ${ }^{\mathrm{c}}$, Y.Y. Wei ${ }^{\mathrm{c}}$, W.S. Hou ${ }^{\mathrm{a}}$ \\ ${ }^{a}$ Department of Physics, National Taiwan University, Taipei, Taiwan \\ ${ }^{\mathrm{b}}$ National Lien Ho College of Tech. and Commerce, Miao Li, Taiwan \\ ${ }^{\mathrm{c} N S T D C}$ of National Tsing Hua University, Hsin-Chu, Taiwan
}

Received 26 November 1999; received in revised form 2 March 2000; accepted 11 March 2000

\begin{abstract}
We measured the radiation damage and its recovery of undoped BGO crystals. A radiation dose of up to $2 \mathrm{Mrad}$ makes the scintillation light output decrease by $20 \%$, but recovers to $100 \%$ with a time constant of $1 \mathrm{~h}$. A radiation dose of $10 \mathrm{Mrad}$ makes the scintillation light output decrease by $30 \%$, and recover to $90 \%$ with a time constant of $10 \mathrm{~h}$. (C) 2000 Elsevier Science B.V. All rights reserved.
\end{abstract}

Keywords: BGO crystals; Radiation damage; Recover time constant

\section{Introduction}

Extreme Forward Calorimeter [1] is under construction for the BELLE detector [2] at KEK Bfactory. The electromagnetic shower medium chosen is Bismuth Germanate $\left(\mathrm{Bi}_{4} \mathrm{Ge}_{3} \mathrm{O}_{12}\right)$, commonly known as BGO. The advantages of the BGO crystals as a shower medium are high density, mechanical stability, relatively short radiation length, large refractive index, fast decay of scintillation light, and excellent $e / \gamma$ energy resolutions. Its radiation hardness is also important because of its proximity to the accelerator beam pipes and the high luminosity of the B-factory.

Our group reported that BGO crystals produced by a certain manufacturer are radiation-hard up to

\footnotetext{
* Corresponding author. Fax: + 886-2-23693472 .

E-mail addresses: ueno@hep1.phys.ntu.edu.tw (K. Ueno).
}

$10 \mathrm{Mrad}$ of $\gamma$ radiation [3]. Our group also reported that the same BGO crystals are considerably radiation-hard up to $90 \mathrm{Mrad}$ and that radiation damage saturates above $10 \mathrm{Mrad}$ [4]. This paper reports on radiation damage and the time dependence of scintillation light output of BGO crystals for low-dose $\gamma$ radiation up to 10 Mrad. When the BGO crystals are exposed to the radiation, the scintillation light output drops temporarily. They recover differently from damage for doses of $10 \mathrm{Mrad}$ obtained in $5 \mathrm{~h}$ and $2 \mathrm{Mrad}$ or less in $1 \mathrm{~h}$. The 10 Mrad dose causes permanent radiation damage.

\section{Experiment setup}

Undoped BGO crystals of trapezoidal shapes were obtained from the Institute of Inorganic Chemistry, Novosibirsk, Russia, originally for 


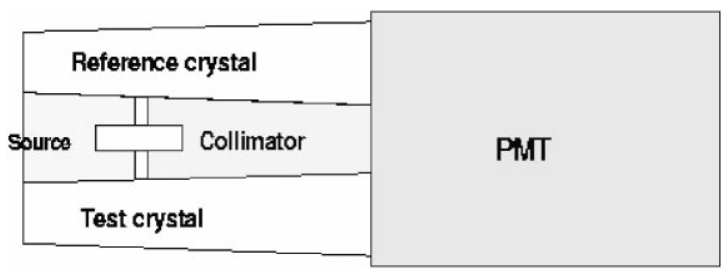

Fig. 1. Schematic diagram of the setup for the scintillation measurement with a ${ }^{60} \mathrm{Co}$ source. Both the reference and the test crystals are exposed to the same source and their scintillation light collected by the same PMT at the same time.

a beam test but also used for this radiation test. The height of the trapezoid is $11 \mathrm{~cm}$ with the top and the bottom surfaces about $0.9 \times 1.4$ and $1.1 \times 1.7 \mathrm{~cm}^{2}$, respectively.

The $\gamma$-ray irradiation was conducted at the irradiation facility of National Tsing Hua University, Hsin-Chu, Taiwan. The source was ${ }^{60} \mathrm{Co}$ with an activity of $1320 \mathrm{Ci}$. Most test crystals were irradiated at their front surfaces except for one crystal. This test crystal was irradiated at the side surface. The former case simulates the real situation in our proposed detector, where the front face of the crystals would point to the interaction point. The test crystals were placed at a distance from the source such that doses of $115 \mathrm{krad}$ and 2 Mrad were obtained in $1 \mathrm{~h}$. A dose of $10 \mathrm{Mrad}$ was obtained in $5 \mathrm{~h}$.

Five test crystals were prepared for the radiation tests. Test crystal 1 was exposed to a dose of $115 \mathrm{krad}$ first. Immediately after the exposure, it was taken out from the radiation cell for measurement of the scintillation light output. The measurement continued for 1 day. After one day test crystal 1 was again exposed to a dose of $2 \mathrm{Mrad}$. After the exposure, the time dependence of the scintillation light output was measured for 4 days. Test crystals $2 \mathrm{a}-\mathrm{d}$ were exposed to a dose of $10 \mathrm{Mrad}$. After the exposure, they were subjected to measurements of the scintillation light output for 8 days.

Before and after a test crystal received its dose, its light output was measured with a ${ }^{60} \mathrm{Co}$ of $89 \mu \mathrm{Ci}$ and a photo-multiplier tube (PMT, Hamamatsu R329-05, 2 in. diameter). A schematic diagram of the setup for the measurement is shown in Fig. 1. A reference crystal and the test crystal were placed side by side and sandwiched a lead collimator of ${ }^{60} \mathrm{Co}$. Both the reference and the sample crystals were viewed and their scintillation light collected by the same PMT at the same time. The setup made possible the measurements of the scintillation light outputs of both the reference and the test crystals at the same time.

The reference crystal was wrapped with Teflon tape to obtain the maximum light collection by the PMT. The test crystal was not wrapped to make its pulse height smaller than that of the reference crystal. The signal from the PMT was digitized with a LeCroy CAMAC ADC 2249A. The ADC system was triggered by self-gate for the scintillation signals produced with ${ }^{60} \mathrm{Co}$ and by a pulser (HP 8112A) for a pedestal. The CAMAC crate was controlled by a PC-based data acquisition system.

Data taken this way show three peaks, separated from each other, corresponding to the signal of the reference crystal, the signal of the test crystal, and the pedestal. Taking the pedestal-subtracted ratio of the signals of the test to the reference crystals, many environmental factors such as temperature and voltages can be cancelled out. We call this ratio as the relative light output.

Immediately after the test crystals received the respective doses, they were taken from the irradiation cell to a nearby experimental booth where the scintillation light output and their time dependence were measured. The light output was measured every $1 \mathrm{~h}$ and the measurement was repeated for 1-4 days. Each measurement took about $15 \mathrm{~min}$. to accumulate 13,000 events.

\section{Result}

The results are a time dependence of the relative light outputs. The relative light outputs are shown in the vertical axis as a function of time of their measurements shown in the horizontal axis. In these figures the first point corresponds to the values prior to the radiation exposure and is normalized to one. The second point is the values measured right after the respective dose. The vertical error bars are statistical. The indicated horizontal error bars in Fig. 2 correspond to $30 \mathrm{~min}$. We 


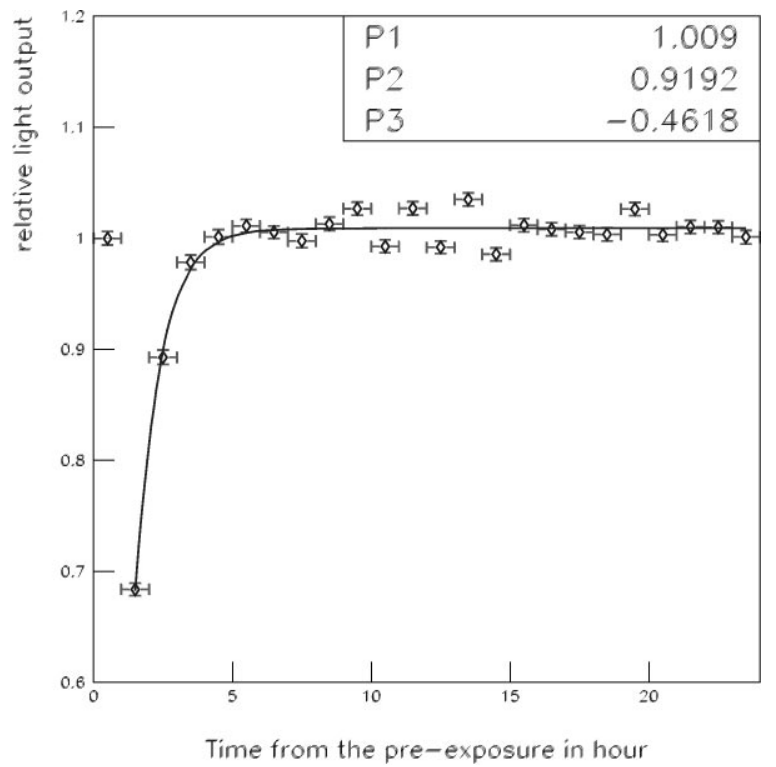

Fig. 2. Test BGO crystal 1 received a dose of $115 \mathrm{krad}$ in $1 \mathrm{~h}$ at its front surface. The time dependence of the relative light output before and after the dose is shown.

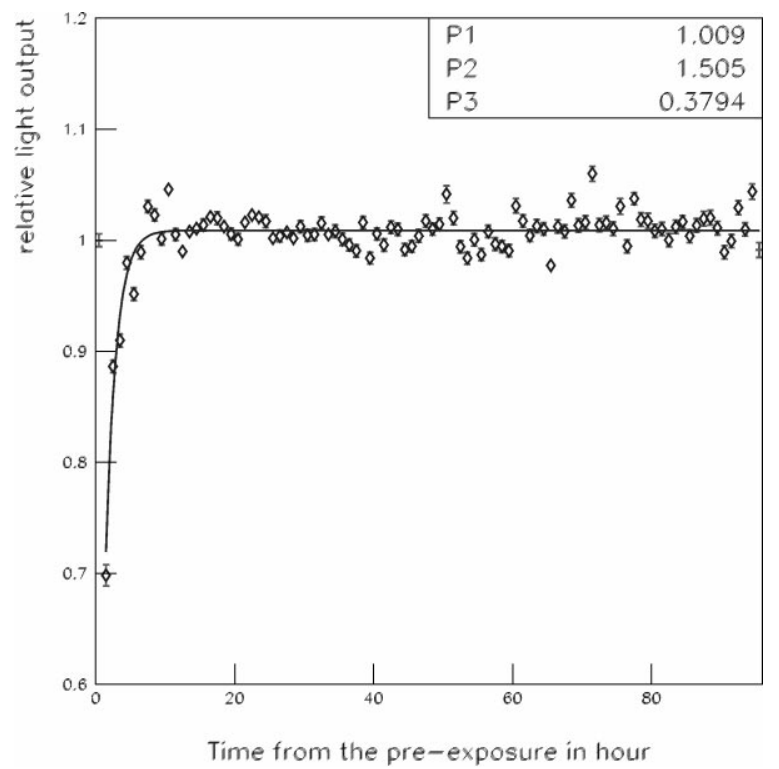

Fig. 3. Test BGO crystal 1 received a dose of $2 \mathrm{Mrad}$ in $1 \mathrm{~h}$ at its front surface. The time dependence of the relative light output before and after the dose is shown.

estimate the systematic error of the relative light output to be $3 \%$ from the fluctuations in the plateau of each figure.

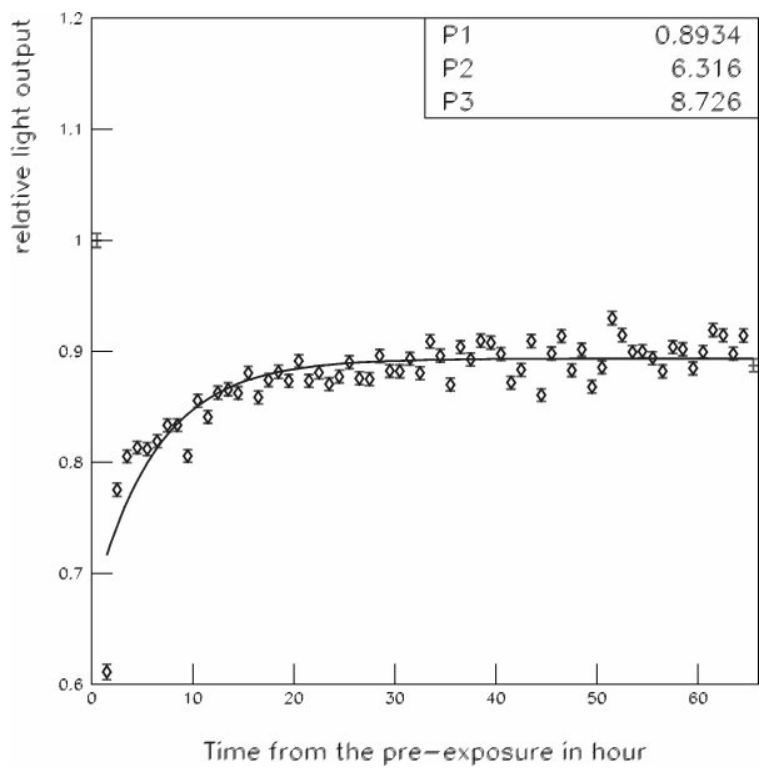

Fig. 4. Test BGO crystal 2a received a dose of $10 \mathrm{Mrad}$ in $5 \mathrm{~h}$ at its front surface. The time dependence of the relative light output before and after the dose is shown.

The results for the doses $115 \mathrm{krad}, 2$ and $10 \mathrm{Mrad}$ on crystals 1, and 2a,b,c,d are shown in Figs. 2, 3, and 4 , respectively. Crystals 1 , and $2 \mathrm{a}, \mathrm{b}, \mathrm{c}$ were exposed to the radiation at their front faces (Figs. 5 and 6). Only the last crystal $2 \mathrm{~d}$ was exposed to the radiation at the side face of the largest surface (Fig. 7).

All these figures show the initial drops of the relative light outputs right after the radiation exposures. The initial drops are from $30 \%$ to $40 \%$ depending on the amount of dose and the crystals. The drops become smaller as time goes by after the radiation exposure and recover to plateaus with some time constants. The recovered parts of the data were fit to a function

$P 1 \times\left(1-\exp ^{-(t+P 3) / P 2}\right)$.

The parameters $P 1, P 2$, and $P 3$ obtained in the fit are shown in each figure. The recover time constants $P 2$ are about 10 and $1 \mathrm{~h}$ for the doses of 10 and 2 Mrad or less, respectively.

Crystals which received the doses of $115 \mathrm{krad}$ and $2 \mathrm{Mrad}$ recover to $100 \%$ of the original relative light outputs before the exposure. Crystals which 


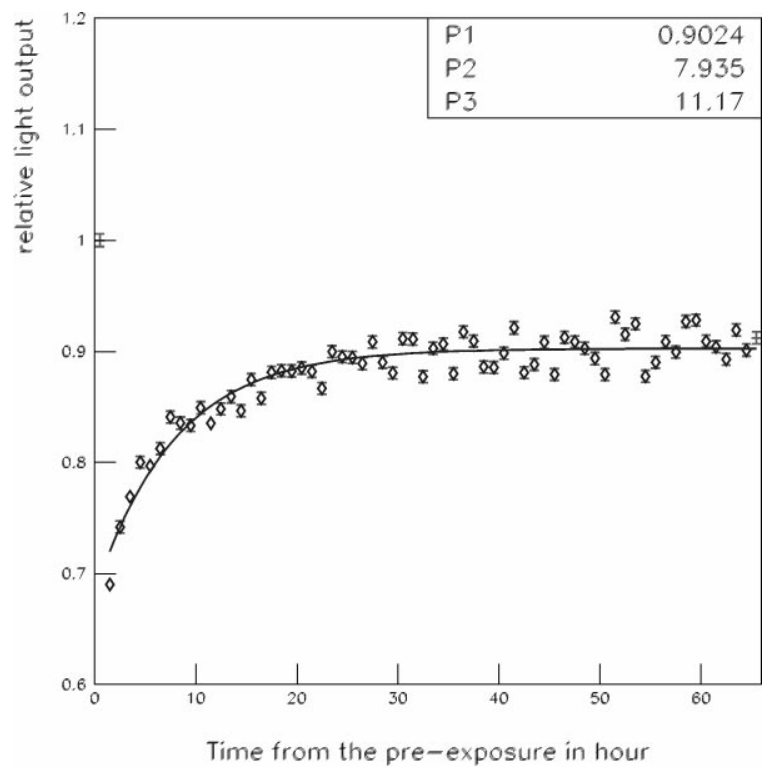

Fig. 5. Test BGO crystal $2 \mathrm{~b}$ received a dose of $10 \mathrm{Mrad}$ in $5 \mathrm{~h}$ at its front surface. The time dependence of the relative light output before and after the dose is shown.

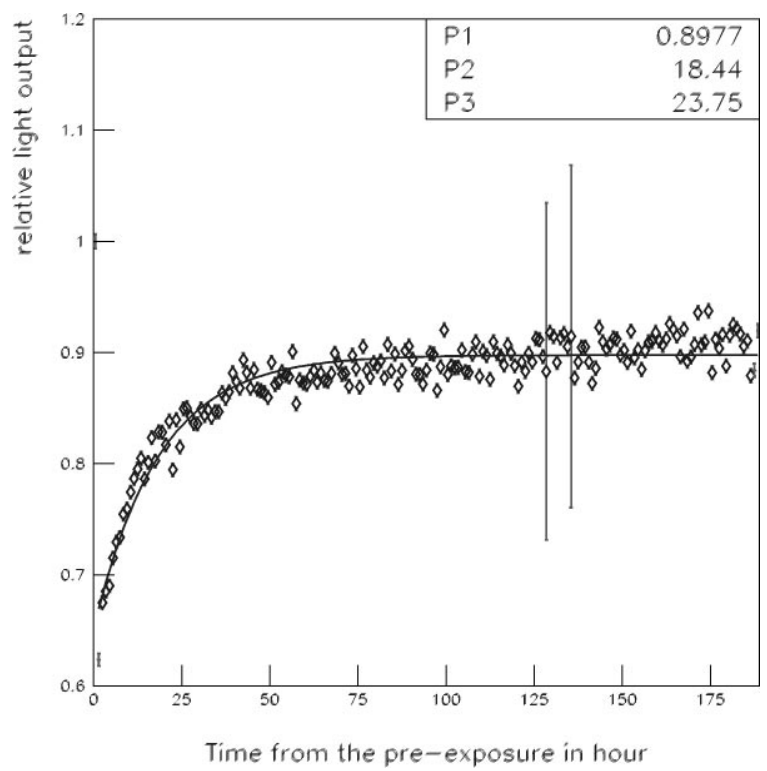

Fig. 6. Test BGO crystal $2 \mathrm{c}$ received a dose of $10 \mathrm{Mrad}$ in $5 \mathrm{~h}$ at its front surface. The time dependence of the relative light output before and after the dose is shown.

received the dose of $10 \mathrm{Mrad}$ recover to $90 \%$ and $97 \%$ for the front exposure and the side exposure, respectively.

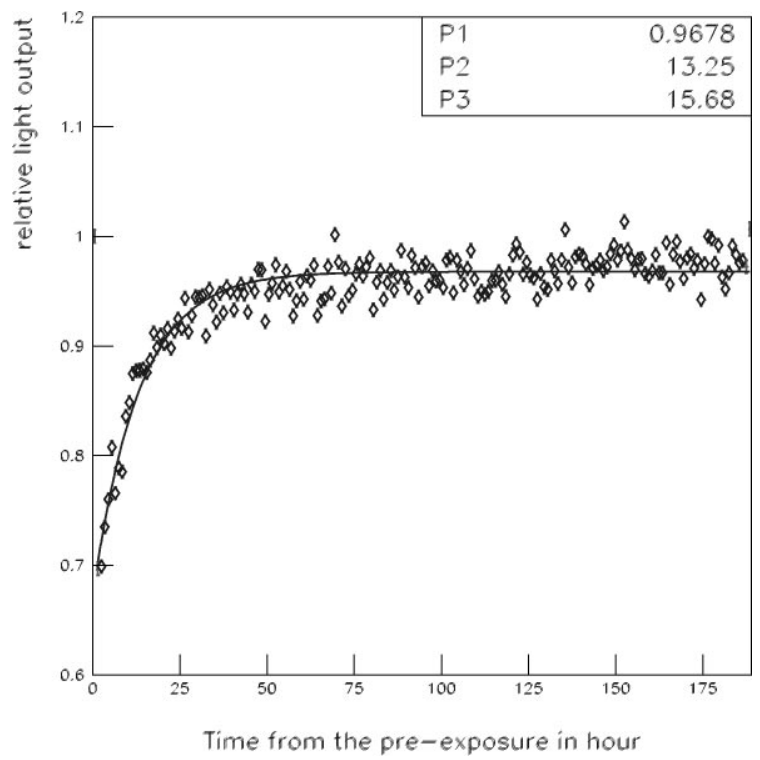

Fig. 7. Test BGO crystal $2 \mathrm{~d}$ received a dose of $10 \mathrm{Mrad}$ in $5 \mathrm{~h}$ at its side surface. The time dependence of the relative light output before and after the dose is shown.

\section{Conclusion}

The crystals get radiation-damaged temporarily with doses of $2 \mathrm{Mrad}$ or less but recover to $100 \%$ of the original with a time constant of $1 \mathrm{~h}$. The crystals get radiation-damaged permanently with doses of 10 Mrad. They recover partially to $90 \%$ of the original with a time constant of $10 \mathrm{~h}$.

\section{Acknowledgements}

We would like to thank the BELLE CsI calorimeter subgroup for its help in this project. We are grateful to the staff members of the irradiation facility at National Tsing Hua University, Hsin-Chu, Taiwan for their help and cooperation. This experiment was partially supported by the Grant NSC 87-2112-M-002-037 of the Republic of China.

\section{References}

[1] W.S. Hou et al., BELLE note 90 (1995).

[2] BELLE Progress Report, KEK Progress Report 97-1 H.

[3] S.K. Sahu et al., Nucl. Instr. and Meth. A 388 (1997) 144.

[4] K.C. Peng et al., Nucl. Instr. and Meth. A 427 (1999) 524. 